http://jmscr.igmpublication.org/home/ ISSN (e)-2347-176x ISSN (p) 2455-0450

crossref DOI: https://dx.doi.org/10.18535/jmscr/v7i11.53

Journal Of Medical Science And Clinical Research

IGM Publication

An Official Publication of IGM Publication

\title{
Outcome of Palliative Fractionated Radiotheraphy in Locally Advanced Inoperable Head and Neck Cancer
}

\author{
Authors \\ Dr Jissy Mathew ${ }^{1}$, Dr Mahadeven $\mathbf{R}^{2}$, Dr Ajith Kumar V R $\mathbf{3}^{*}$, Dr Shehna A Khader \\ Dr Jewel Joseph \\ ${ }^{1}$ Senior Resident, ${ }^{2}$ Professor, ${ }^{3}$ Assistant Professor, ${ }^{4}$ Associate Professor, ${ }^{5}$ Senior Resident \\ *Corresponding Author \\ Dr Ajith Kumar V R
}

Assistant Professor, Department of Radiation Oncology, Government Medical College Chest Hospital,

Thrissur, India

\begin{abstract}
Background and Objectives: Hypofractionated radiotherapy has been used for palliation in locally advanced inoperable head and neck cancer patients. A cyclical hypofractionated regime RTOG 8502 originally used for palliation in advanced pelvic malignancy has proved to be effective in palliation of locally advanced inoperable head and neck cancer. Radiation is delivered in 2 fractions of 3.7 gray per day at 6 hrs interval on 2 consecutive days with cycles repeated at 4 weeks gap. A prospective observational study was done in radiotherapy department Government medical college Thrissur with the primary objective of assessing the palliative response when RTOG 8502 regime is used in patients with locally advanced inoperable head and neck cancer. Secondary objectives of the study were to assess the quality of life, tumor response, toxicity and overall survival.

Methods: 51 patients who satisfied inclusion criteria and included in the study population was treated with palliative radiation using RTOG 8502 regime (3.7 gy twice daily at 6 hours interval over 2 consecutive days repeated at 4 weeks).

Conclusion: Palliative hypofractionated radiotherapy using RTOG 8502 regime (quad shot) is an effective palliative treatment modality in locally advanced inoperable head and neck cancer. With excellent palliative response, radiobiological effect sparing normal tissue toxicity and improved overall survival it offers a better quality of life at low cost, best suited for centres with crowded population and poor patient compliance.

Keywords: Locally advanced head and neck cancer-Palliative fractionated radiotherapy -Palliative response-quality of life.
\end{abstract}

\section{Introduction}

Head and neck cancer is the $6^{\text {th }}$ leading cancer worldwide. There are 5 million cases of head and neck cancer reported each year. ${ }^{1} 60 \%$ of these cancer present in locally advanced non metastatic stage .In India there is approximately 250000 head and neck cancer reported each year.90\% of the head and neck cancers are squamous cell carcinoma involving lip, oral cavity, larynx, oropharynx, nasopharynx, hypopharynx and paranasal sinus. Alcohol and tobacco use are the common etiological factors associated with head and neck cancer. Other risk factors include Ebstein Barr Virus and Human Papilloma Virus 
infection. ${ }^{1,2}$ The number of patients with HPV positive oropharyngeal cancer is increasing each year ${ }^{3}$, commonest site of involvement being the tonsillar fossa and the base of tongue.

Head and neck cancer patients have some pre morbid features that make them a challenging group compared to patients with cancer at other sites. They have a tendancy to abuse tobacco or alcohol or both and are less meticulous in personal habits especially oral hygiene. They usually have poor nutrition and mostly belong to low socioeconomic status. Most of the patients with head and neck cancer usually present with locally advanced disease irrespective of metastasis.

In India, about $70-75 \%$ cases of head and neck cancer patients present in a locally advanced stage with a significant proportion in an inoperable state. $^{2}$ Due to poor general condition, comorbidities and extensive loco regional involvement curative treatment is not possible in most cases. A large proportion of these cases are surgically inoperable due to invasion of carotid space, prevertebral fascia or extension to the infratemporal fossa. The patient will present with intractable pain, tumour hemorrhage or asphyxiation. The intent of treatment of these cases is mainly palliative, offering symptom relief thus improving the physical and psychosocial wellbeing of the patient and giving a better quality of life.

Head and neck cancers need a multidisciplinary approach for management especially in advanced disease. Head and neck is an area where critical structures like spinal cord and brainstem are in close proximity to tumor bearing area. Also there is high chance for morbidity because the subsites in head and neck are involved in critical functions like speech, swallowing and breathing. So the functional outcome should also be considered when deciding the treatment modality in radical or palliative approach.

In patients with locally advanced inoperable head and neck cancer with good performance status and no comorbidities concurrent chemoradiation can be given .In inoperable head and neck cancer patients with comorbidities, metastatic disease and in those patients who cannot tolerate chemotherapy due to toxicity radiotherapy has been demonstrated to be an effective palliative modality .

Hypofractionated radiotherapy regimens have been extensively used for palliation in view of better patient compliance. Different fractionation schedules offering good palliative response with limited toxicity have been studied.

A cyclical hypofractionated palliative radiotherapy regimen originally devised for advanced pelvic malignancies by radiotherapy and oncology group RTOG8502 $2^{4,5,6}$ using $14.8 \mathrm{~Gy}$ in 4 fractions has been successfully adapted for palliation of inoperable locally advanced and metastatic head and neck cancer. ${ }^{7}$ It was found to have less toxicity, better symptomatic relief and quality of life. Other advantages were the ease of delivery in a simple clinical setup at low cost. ${ }^{8}$ In comparative studies patients receiving radiation in fractionated doses using RTOG 8502 regimen had a better side effect profile and median overall survival of up to 5.67 months compared to other treatment groups. ${ }^{8}$ The tumour response rate was $77 \%$ and palliation 65 to $85 \%$ as assessed by patient report ${ }^{5,7,9}$ Grade 3and 4 toxicity were significantly low compared to conventional radiation regimenn., ${ }^{7,9}$ The palliative response related to symptoms like odynophagia and dysphagia was $53 \%$ and for trismus and epistaxis was $100 \%$ in some observational studies. ${ }^{9}$ Patients receiving more number of cycles of fractionated RTOG8502 Regimen had better overall survival rates. ${ }^{7,9}$ Other recommended palliative fractionation regimens are 50 Gray in 20 fractions, 37.5 Gray in 15 fractions, 30 Gray in 10 fractions, 30 Gray in 5 fractions.

In our institution we have an average of 4000 patients registered with cancer each year. According to ICMR data we had 4009 new cancer cases registered in 2017 of which head and neck cancer constituted about 700 cases. About 400 of those cases were in advanced stage that is stage 3 and 4 with stage 4 accounting for $75 \%$ of cases. 
Locally advanced inoperable and metastatic disease was present in 75 patients. Our intent of treatment in such patients is to give maximum palliative benefit with minimum toxicity. We routinely use the palliative regimen of $14.8 \mathrm{~Gy}$ in 4 fractions based on RTOG 8502. Radiation is given as 3.7 Gray fractions, 2 fractions per day for 2 days repeated at 4 weekly interval for 3 cycles to these advanced head and neck cancer patients. Since this regimen has low toxicity and avoids prolonged hospitalization we are able to get better patient compliance and tolerance. This study aims to highlight the benefit of the 14.8/4 regimen otherwise called the Quad shot regimen in palliation of metastatic and locally advanced inoperable head and neck cancer.

\section{Objectives of the Study}

The primary objective is

To prospectively evaluate the palliative response of the patients with advanced head and neck cancer after palliative hypofractionated radiotherapy.

Secondary objectives are
1. To assess the quality of life of the patients before, during and after radiotherapy as per EORTC
2. To assess toxicity profile (RTOG)
3. To assess overall survival

\section{Materials and Methods}

Study Design: prospective/ observational study.

Study Setting: Hospital based observational study will be conducted in the Department of Radiotherapy, Medical College Chest Hospital, Thrissur.

\section{Study Population}

All the patients with advanced and metastatic head and neck cancer attending radiotherapy op unfit for curative treatment will be taken for study

\section{Inclusion Criteria}

1) Histopathologically proven advanced head and neck cancer

2) Surgically inoperable, locally advanced and metastatic cancer ( Stage IV B and C)
3) Stage IVA disease with poor performance status or significant comorbid illness

4) No previous history of radiotherapy within 6 months

5) ECOG status 0 to 3 , mentally competent to give informed consent

\section{Exclusion Criteria}

1) Non consenting patients

2) Early stage carcinoma curable by surgery

3) Active infection

\section{Study Period}

One and a half years from the date of ethical committee clearance

\section{Sample Size}

Sample size is calculated using the formula $\mathrm{n}=$ $3.84 \mathrm{pq} / \mathrm{d}^{2}$ where $\mathrm{p}=\%$ of patients who achieved the target that is palliative response in an RTOG 8502 TRIAL conducted by Benjamin lok etal.q=100-p,. $d=20 \%$ of $p$, considering $p=65$, $\mathrm{n}=51$.

\section{Study Tools}

Specially designed Performa to collect sociodemographic and clinical variables

CT Neck /USG Neck, chest x ray for assessing local and systemic spread

ECOG Performance scale graded o to 5 from fully active to dead

Symptom check list

EORTC QLQ30 quality of life questionnaire developed by European organization for research and treatment of cancer for assessing physical psychological and social function of cancer patients. It has high reliability and validity Toxicity scale (RTOG) graded 1to 4 based on local skin and mucous membrane reaction

QLQ H\&N35 questionnaire assessing quality of life in head and neck cancer patients graded from 1 to 4 depending on the local effect of the tumor in head and neck area and symptomatic relief after radiation.

\section{Methodology}

People who meet inclusion criteria will be made to fill a symptom checklist of 9 items (appendix 1) 
before start of radiation which comprise of 9 entities related to local effect of tumour spread.

Performance status of patients will be assessed by ECOG performance scale (appendix 2)

All the patients included in the study will be given radiation using telecobalt machine by $2 \mathrm{D}$ planning in hypofractionated dose that is 2 fractions of 3.7 Gy at 6 hrs interval per day for 2 consecutive days. A total radiation dose of 14.8 grey in 4 fractions are thus given to the patient. This cycle is repeated at 4weeks interval for a total of 3 cycles Patients will be assessed clinically during RT and after each cycle of radiation at 2 weekly interval till the completion of radiation treatment. After that, monthly follow up is done. If grade 3 or 4 toxicity or progression develops in between the cycles radiotherapy is stopped. After completion of 3 cycles of radiotherapy, chemotherapy or surgery is planned depending upon treatment response and disease status.

Response of tumour is noted at the end of each cycle, after 3 cycles and on follow up using Recist criteria (appendix 3) Radiological assessment of tumour is done by ultrasound or CT scan.

The patients are asked to answer the symptom checklist before, during, after completion 3 cycles of radiation and on monthly follow up. Performance status before, during and after Radiotherapy are compared.

Quality of life of patients before, during and after Radiotherapy will be assessed using EORTC Questionnaire (appendix 4)

Head and Neck symptomatic assessment by QLQ H\&N35 (appendix 5)

Local reaction at radiation site assessed by RTOG toxicity scale (appendix 6)

Overall survival is taken from date of starting first cycle of radiation till last follow up or death

\section{Data Analysis}

Descriptive analysis and logical regression.

\section{Ethical Aspects}

The study will be conducted only after the approval and clearance from Ethical committee, Govt Medical College, Thrissur.
Written informed consent will be obtained from each study participants and all patient details will be kept confidential.

\section{Results}

Among the Head and neck cancer patients with locally advanced inoperable disease registered in department of radiotherapy 51 patients satisfying all inclusion criteria were taken up for study with their consent in the $1.5 \mathrm{yr}$ of study period.

\section{Patient Characteristics}

Age

Table 1: Distribution of Age

\begin{tabular}{|l|c|c|}
\hline Age (Years) & Frequency & Percent \\
\hline Up to 50 & 9 & $17.6 \%$ \\
\hline $51-60$ & 17 & $33.3 \%$ \\
\hline $61-70$ & 15 & $29.4 \%$ \\
\hline $71-80$ & 10 & $19.6 \%$ \\
\hline
\end{tabular}

The mean age of the study population was 50 to 60 yrs ranging from 40 to $80 \mathrm{yrs}$.Majority of the patients were 51 to $60 \mathrm{yrs}(33.3 \%)$ and 61-70yrs (29.4\%).Less than $50 \mathrm{yr}$ age group constituted $17.6 \%$ and 71 to $80 \mathrm{yr}$ age group constituted $19.6 \%$ of the study population

\section{Gender}

Table 2: Distribution of Sex

\begin{tabular}{|l|c|c|}
\hline Sex & Frequency & Percent \\
\hline Male & 34 & $66.7 \%$ \\
\hline Female & 17 & $33.3 \%$ \\
\hline
\end{tabular}

Percentage of Male patients included in study population was 66.7 almost twice the number of females (33.3)

\section{Stage of Disease}

$52.9 \%$ had stage 4 A diseases with comorbidities. $45.1 \%$ had stage $4 \mathrm{~B}$ disease and $2 \%$ had metastatic disease.

Table 3: Distribution based on T stage

\begin{tabular}{|c|c|}
\hline T Stage & Percent \\
\hline Tx & $2.0 \%$ \\
\hline T2 & $7.8 \%$ \\
\hline T3 & $13.7 \%$ \\
\hline T4A & $45.1 \%$ \\
\hline T4B & $31.4 \%$ \\
\hline
\end{tabular}




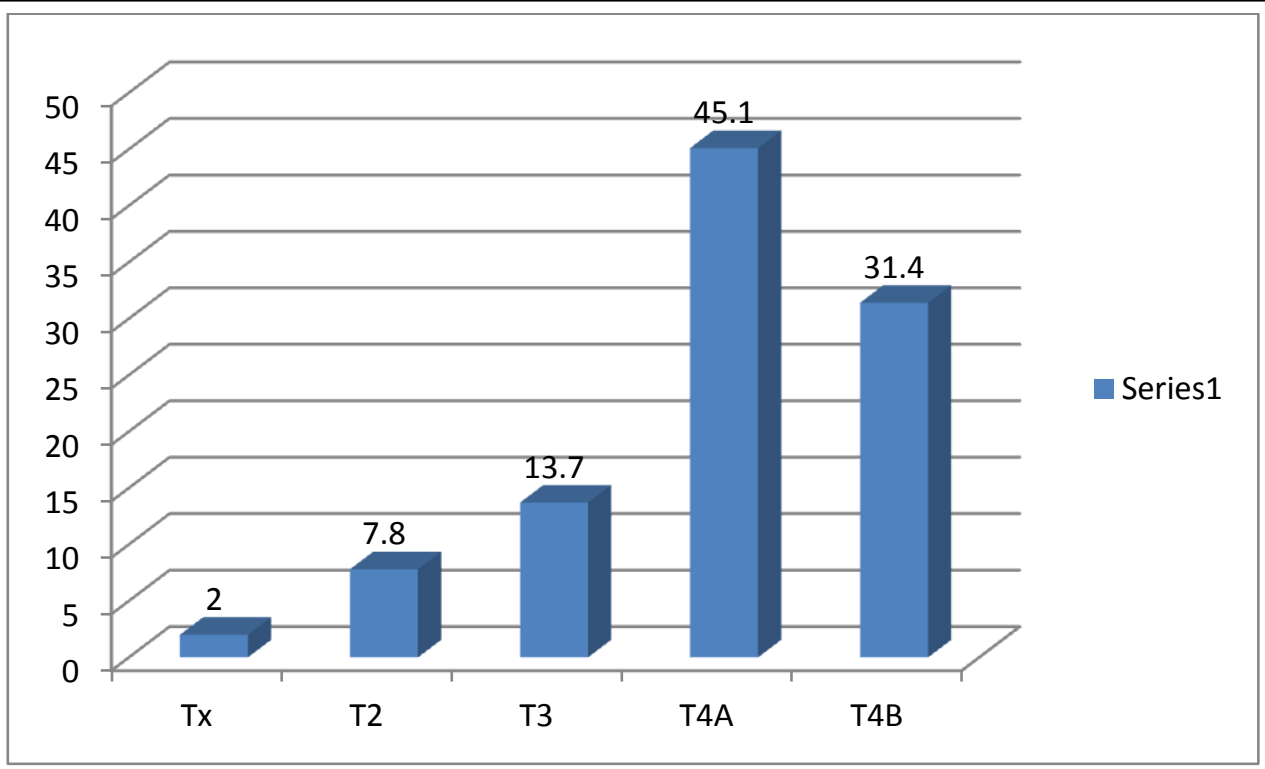

Figure 1 Majority of the cases were T4A (45.1\%) andT4B (31.4\%) respectively

\section{Histopathology}

98\% of the cancer patients included in the study group were squamous cell carcinoma. $2 \%$ had mucoepidermoid tumor

$45.1 \%$ were smokers, $27.5 \%$ had tobacco chewing history, smoking with alcohol both were seen

\section{Palliative Response}

\section{Pain}

There was significant improvement in pain response with each successive cycle of radiation
In patients completing only 2 cycles of radiation the palliative response was less than those completing 3 cycles of radiation. Overall $86 \%$ of the patients had symptomatic relief of pain. In patients completing 3 cycles of radiation $95 \%$ got palliative response to pain. $70 \%$ of non responders belonged to group of patients taking Only 2 cycles of radiation.

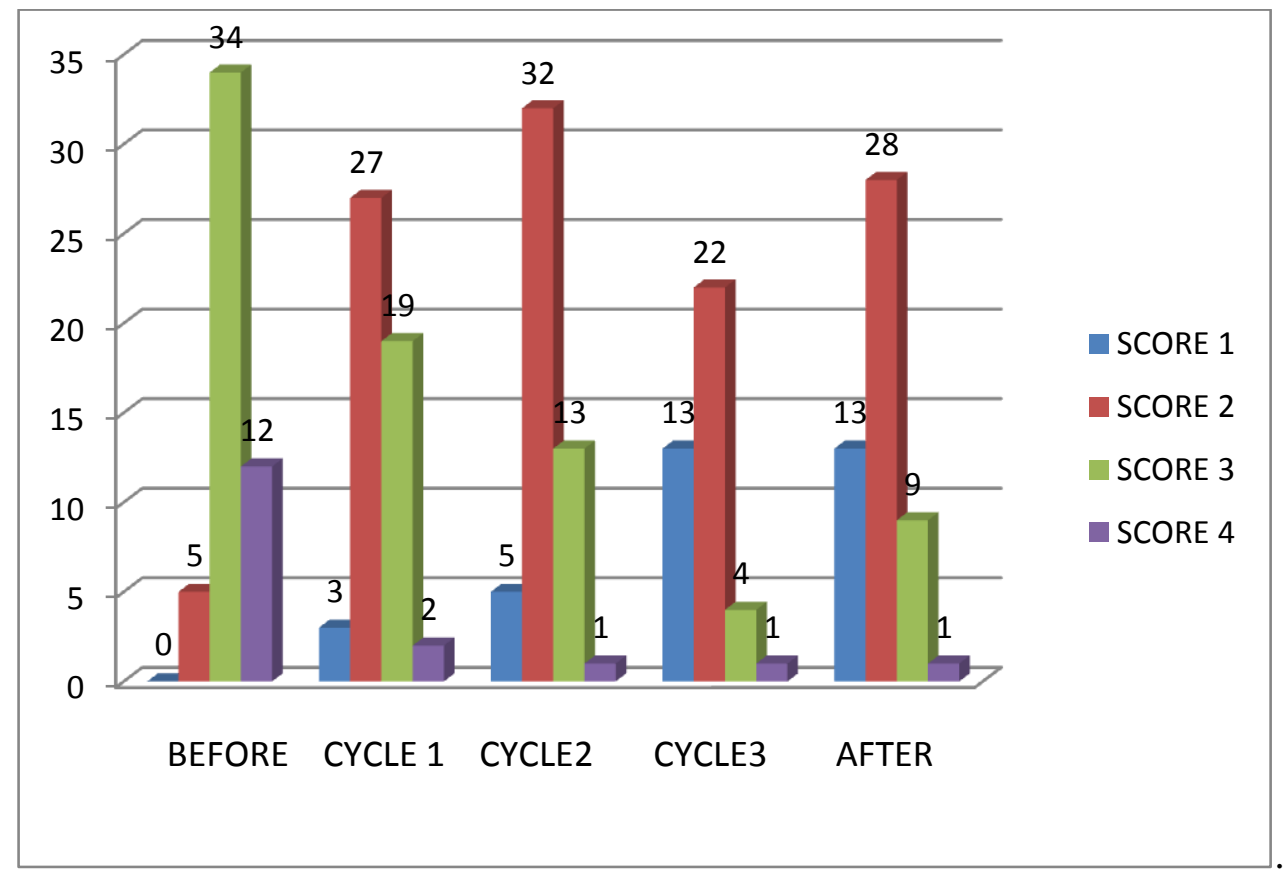

Figure 2: Palliative response to pain 


\section{JMSCR Vol||07||Issue||11||Page 307-318||November}

\section{Trismus}

Trismus was present in 27 patients before radiotherapy.85 \% of these patients had a palliative response to trismus there was significant improvement in trismus with each cycle of radiation Among the study population only $78.4 \%$ completed 3 cycles of radiation. Symptomatic response was more in patients taking 3 cycles of radiation compared to those who had only 2 cycles of radiation.

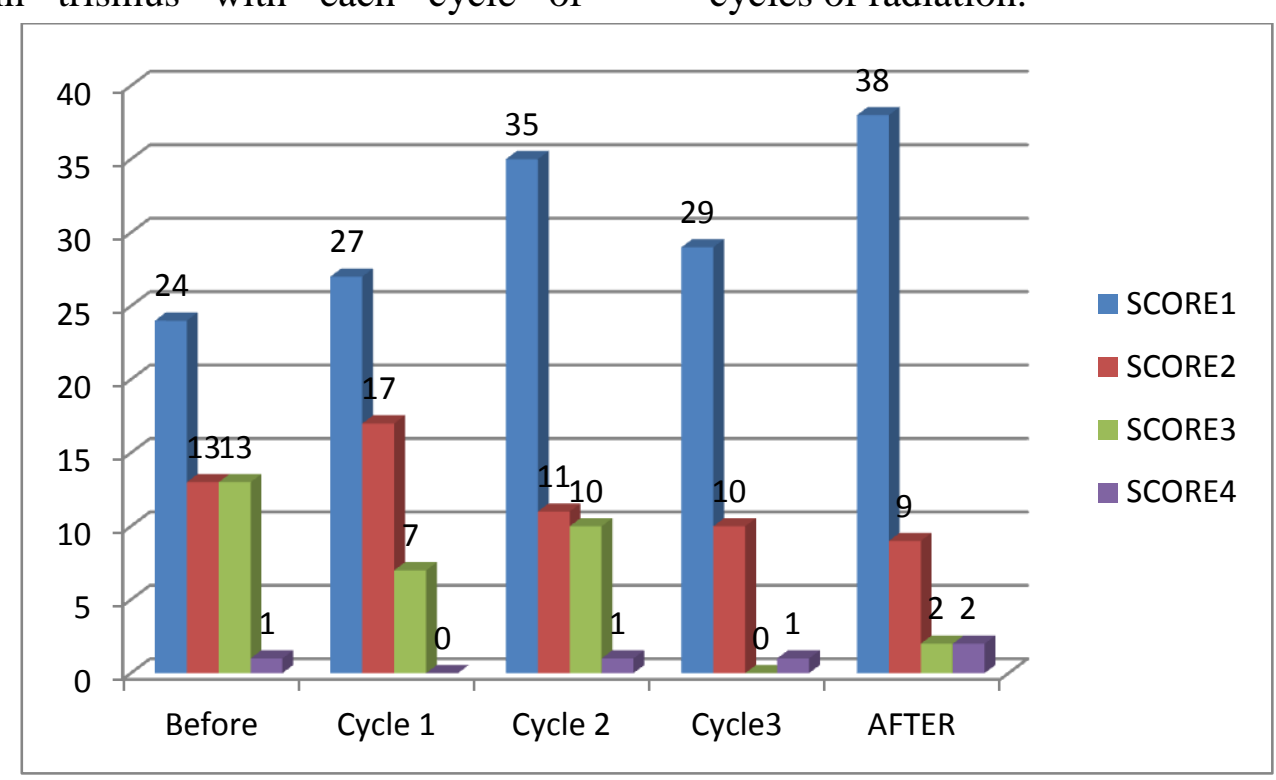

Figure 3 Palliative response to trismus

\section{Dysphagia}

There was an appreciable improvement in dysphagia by improvement in dysphagia after 2 cycles of radiation when compared to the initial presentation. The number of patients with moderate and severe dysphagia was reduced from
$54.9 \%$ to $13.7 \%$ and $13.7 \%$ to $5.9 \%$ respectively.78.4\% of patients had a palliative response to dysphagia. However patients who had 3 cycles of radiation had better palliative response.

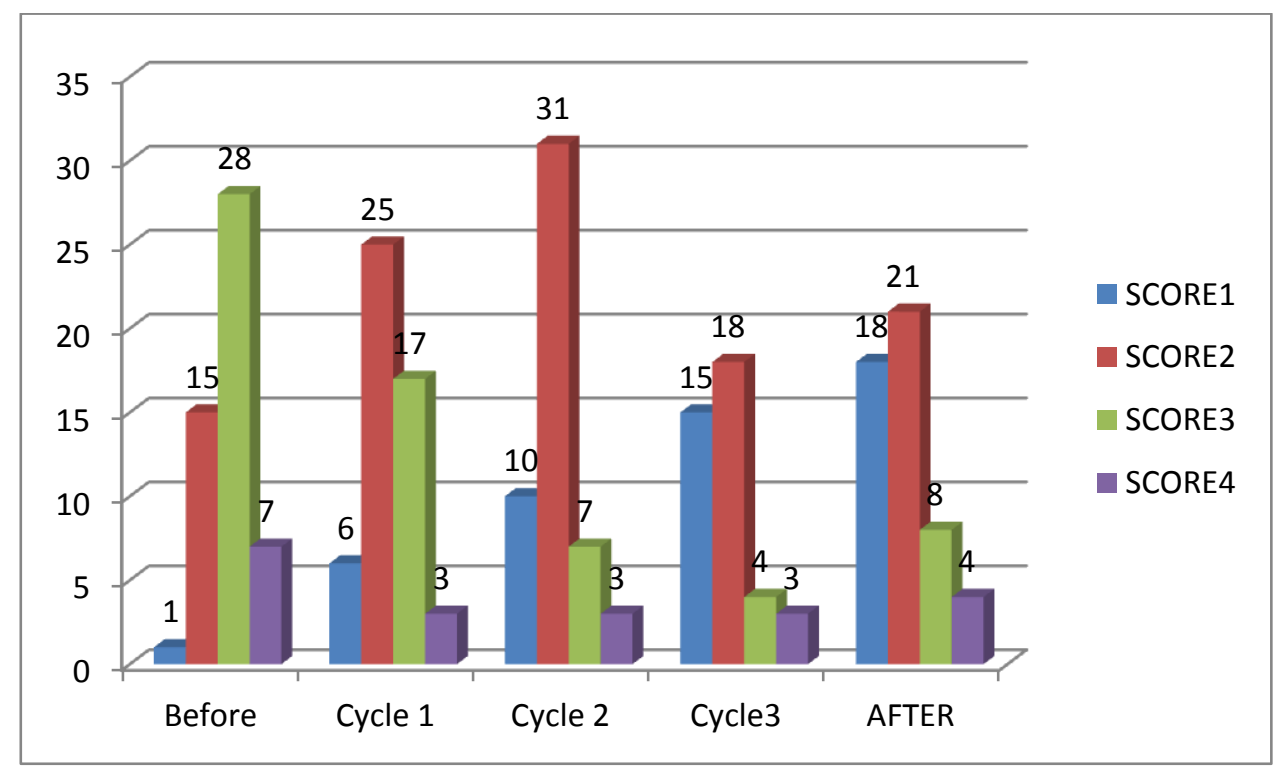

Figure 4 Palliative response to dysphagia 


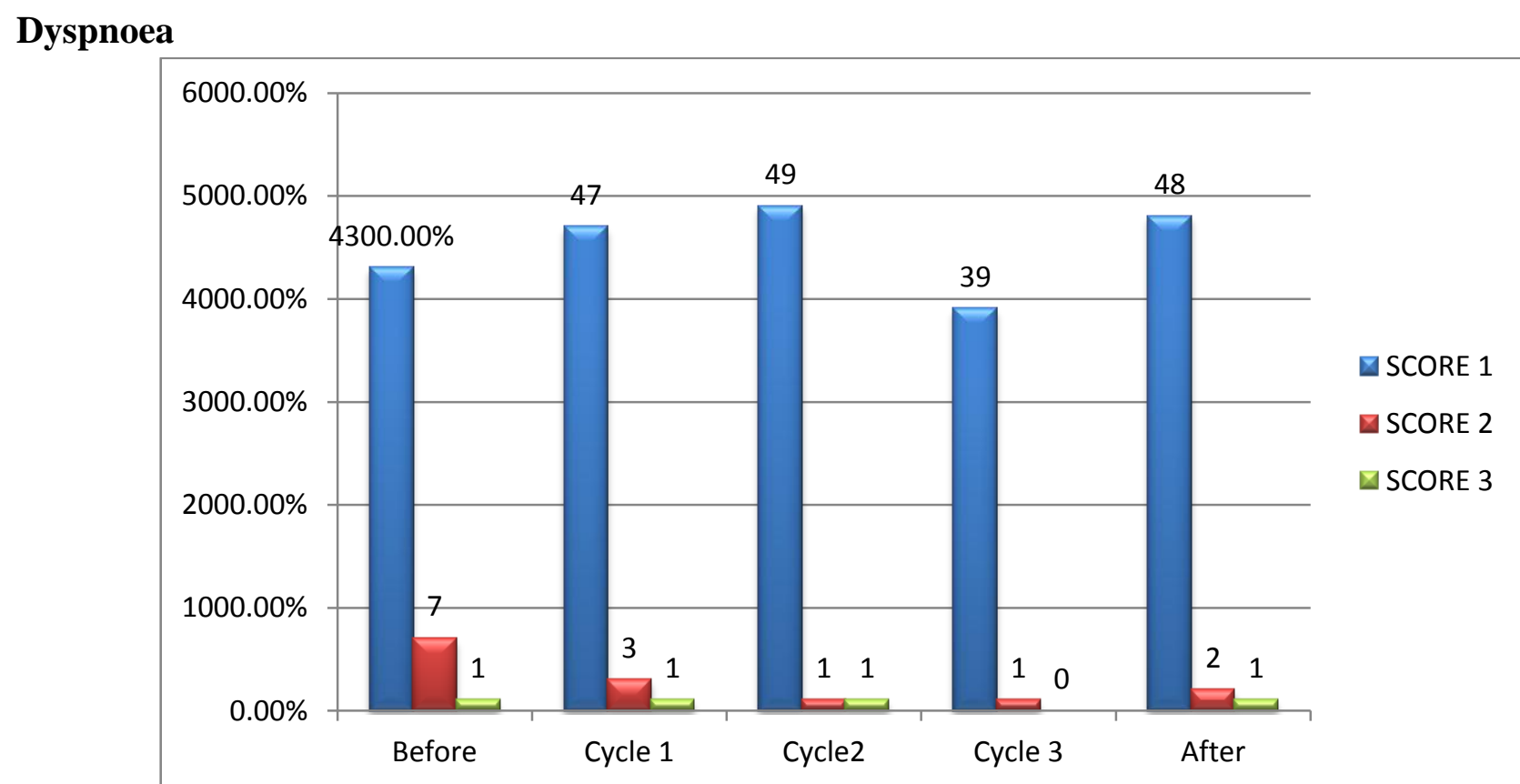

Figure 5: Palliative response to dyspnoea

Dyspnoea was a presenting symptom in only 8 patients $(15.7 \%)$ out of 51 included in study population. 5 out of these $(62.5 \%)$ patients had a symptomatic improvement following radiation. Post radiation only 3 patients complained of dyspnoea. There was no appreciable difference in palliative response in cycle 3 compared to cycle 2 .

\section{Stridor}

Stridor was present in only3 patients (5.9\%)before radiation. After 1 cycle of radiation there was only $2 \%$ left with stridor. After 2 cycles there was complete palliative response.

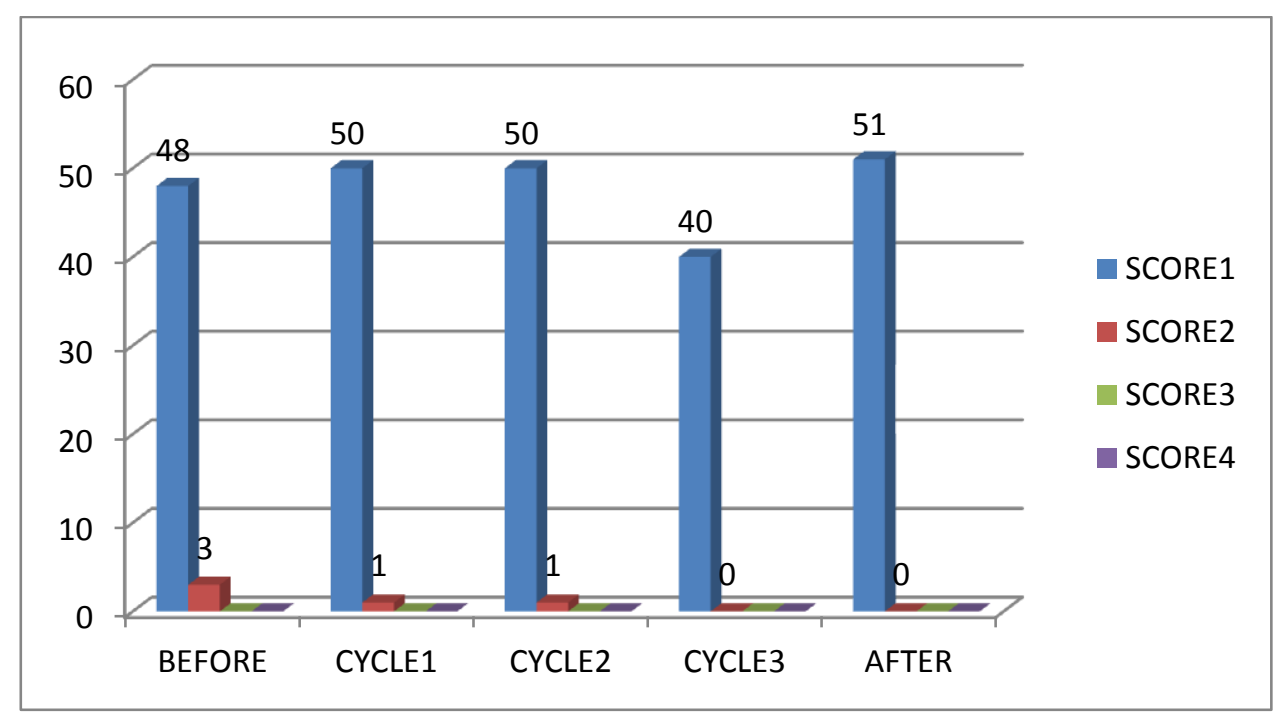

Figure 6 Palliative response to stridor

\section{Bleeding}

Bleeding was present from primary tumor site or neck node in 20 patients $(39.2 \%)$ included in study population. After 1 cycle of radiation 10 patients had complete response to bleeding. After
2 cycles of radiation 7 more patients $(94.2 \%)$ had complete response to bleeding. After completing 3 cycles of radiation none of the patients had bleeding. 


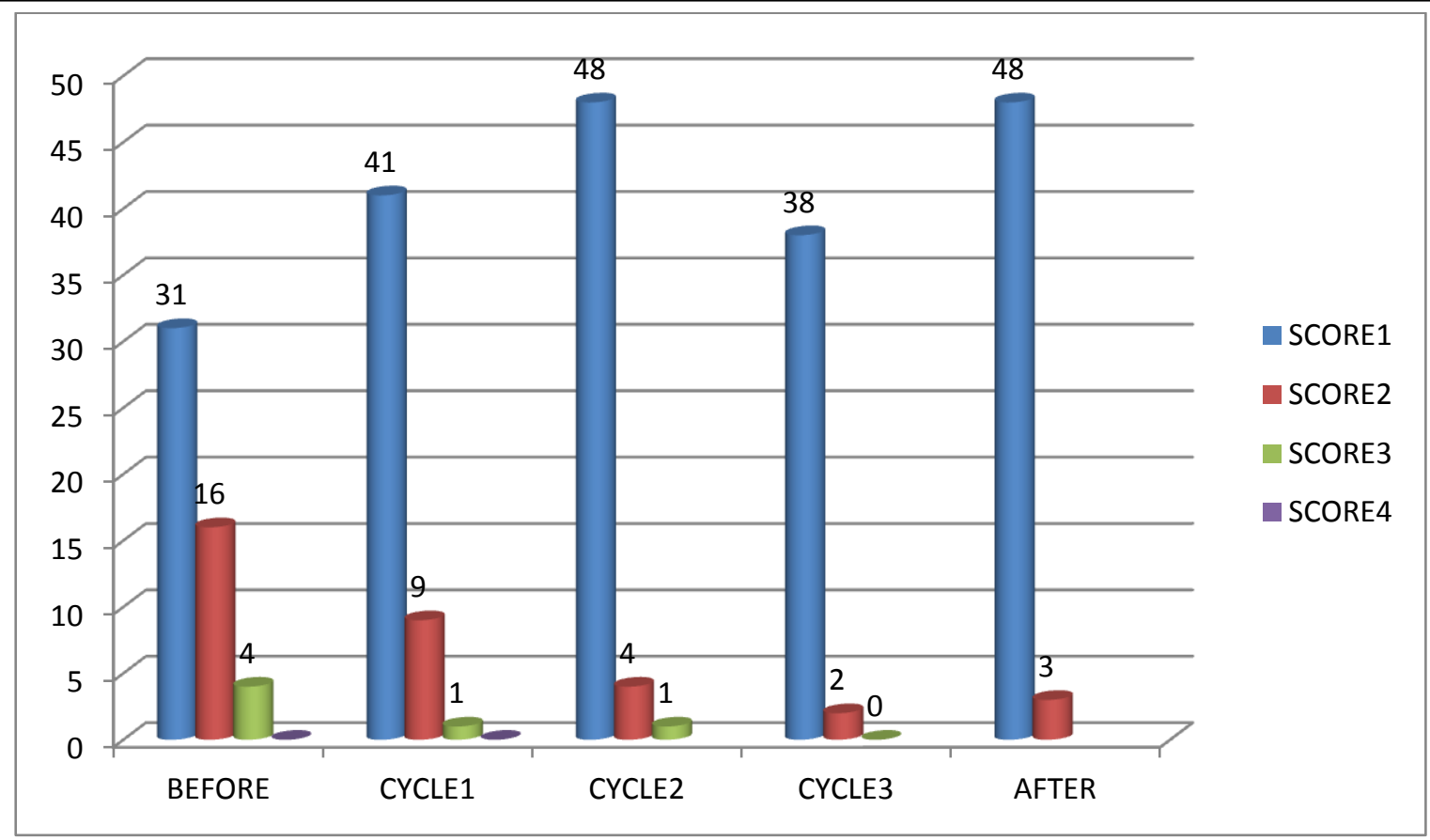

Figure 7 Palliative response to bleeding

\section{Epistaxis}

Only 4 patients $(7.8 \%)$ in the study population complained of epistaxis at presentation. After first cycle of radiation 1 patient had complete response. After 2 cycles of radiation1 more patients had complete response. On completion of 3 cycles of radiation $50 \%$ of the patients with epistaxis got complete response to radiation.

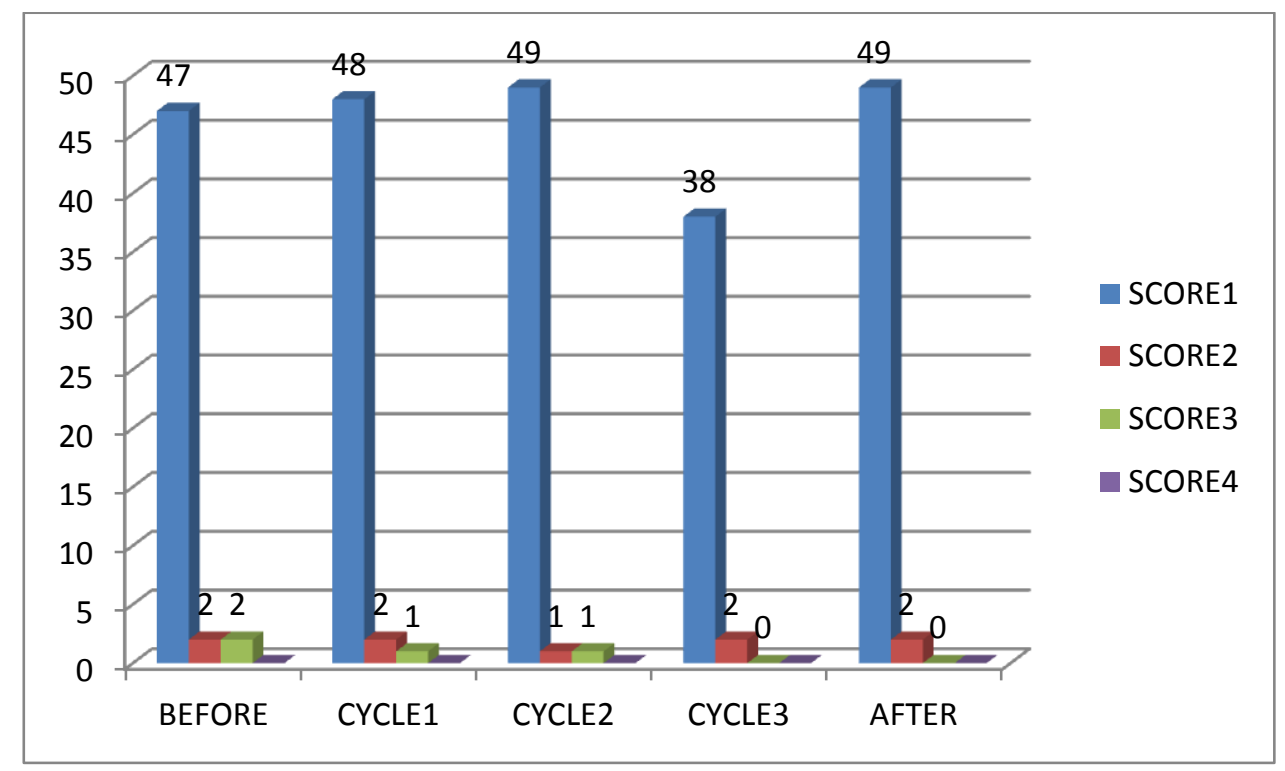

Figure 8 Palliative response to epistaxis

\section{Tumor response}

Tumor response was assessed after each cycle and at the end of 3 cycles. All patients included in the study had a static disease after first cycle. After second cycle $80.4 \%$ had partial response. After 3 cycles complete response was seen in 5 patients.
On follow up after radiation $7(13.7 \%)$ of the 51 patients had complete tumor response. Tumor response was more in patients who received 3 cycles of radiation. 4 patients $(7.8 \%)$ developed progression of disease after radiation, 2 were among those who took 2 cycles of radiation. 


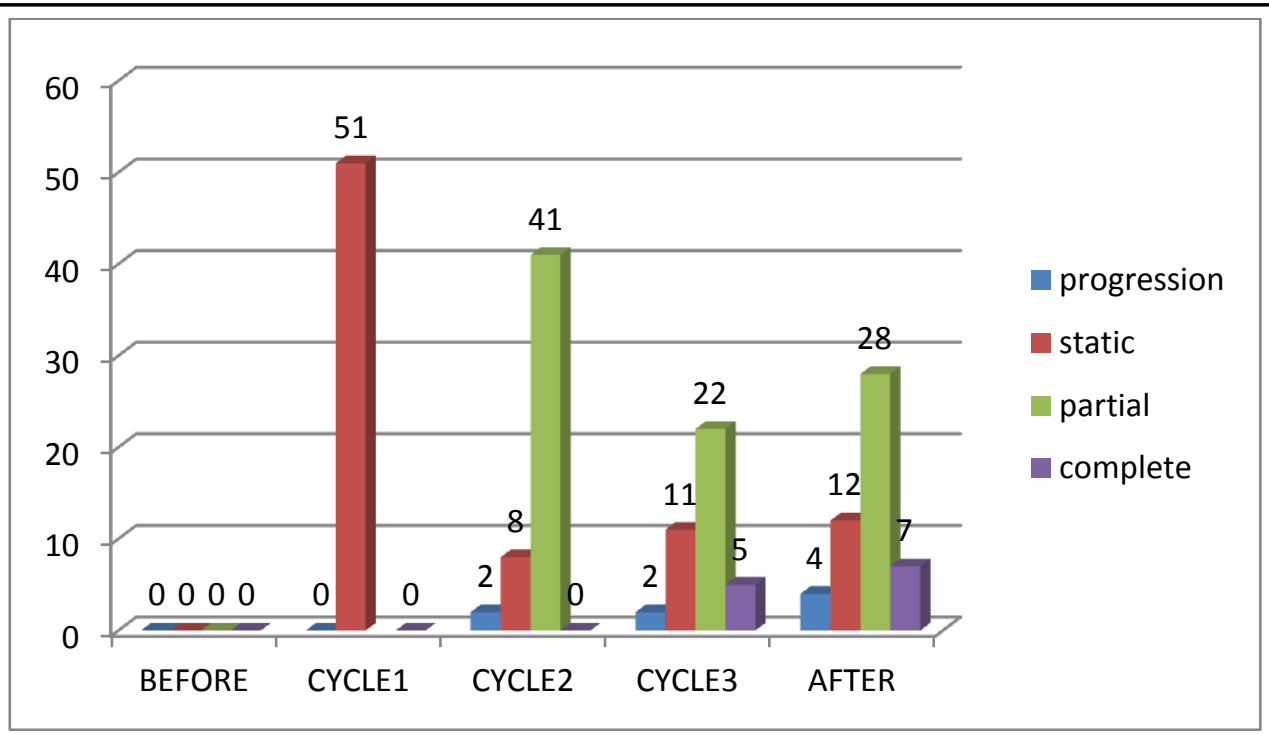

Figure 9: Tumour Response

\section{Overall Survival}

Table 4 Overall survival in months

\begin{tabular}{|l|c|c|}
\hline Overall Survival (Months) & Frequency & Percent \\
\hline $1-3$ & 15 & $29.4 \%$ \\
\hline $4-6$ & 19 & $37.3 \%$ \\
\hline $7-12$ & 10 & $19.6 \%$ \\
\hline$>12$ & 7 & $13.7 \%$ \\
\hline
\end{tabular}

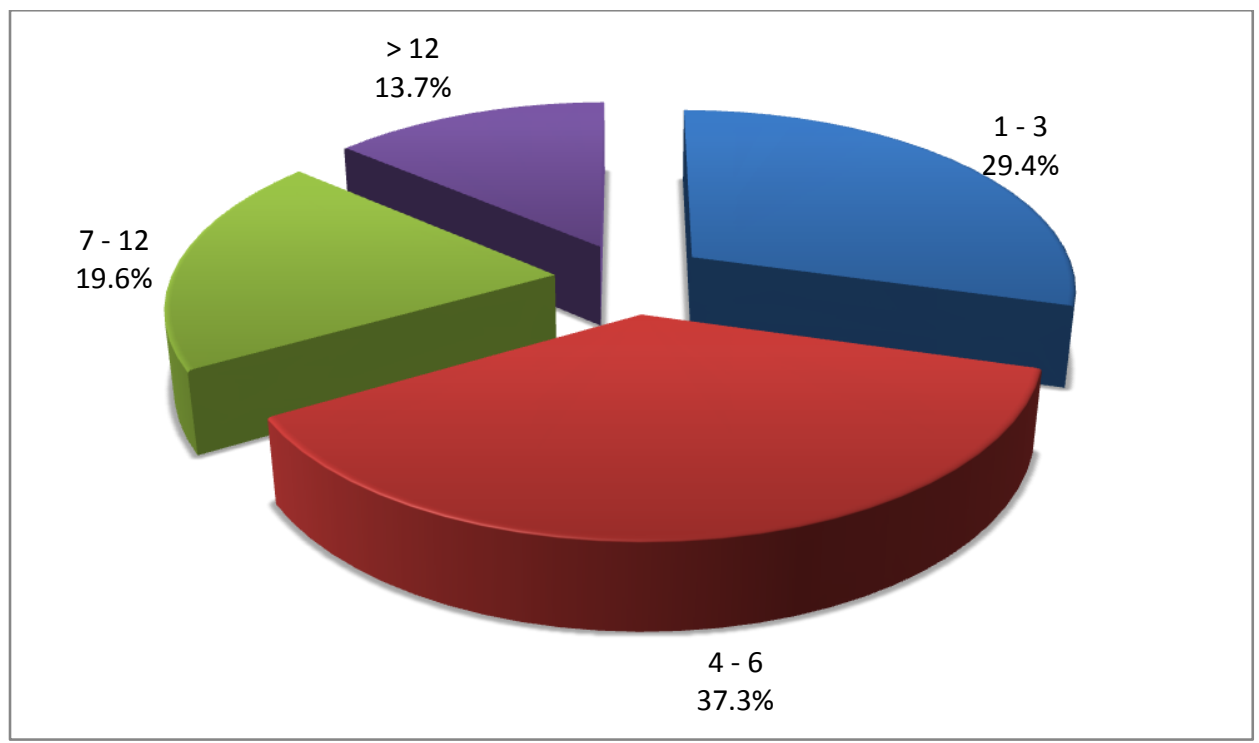

Figure 10 Overall survival in months

Table 5 Comparison of duration of survival among those received 2 cycles and those received 3 cycles

\begin{tabular}{|l|c|c|c|c|c|}
\hline No of cycles received & $\mathrm{N}$ & Mean & Std. Deviation & $\mathrm{t}$-value & $\mathrm{p}$-value \\
\hline 2 & 11 & 3.00 & 1.095 & \multirow{2}{*}{5.647} & $<0.001$ \\
\hline 3 & 40 & 7.25 & 4.277 & & \\
\hline
\end{tabular}

Mean survival among those patients who took 2 cycles of radiation was 3 months and those who received 3 cycles of radiation were 7 months.
Some patients who have received 3 cycles of radiation have survived for more than $1.5 \mathrm{yrs}$. 


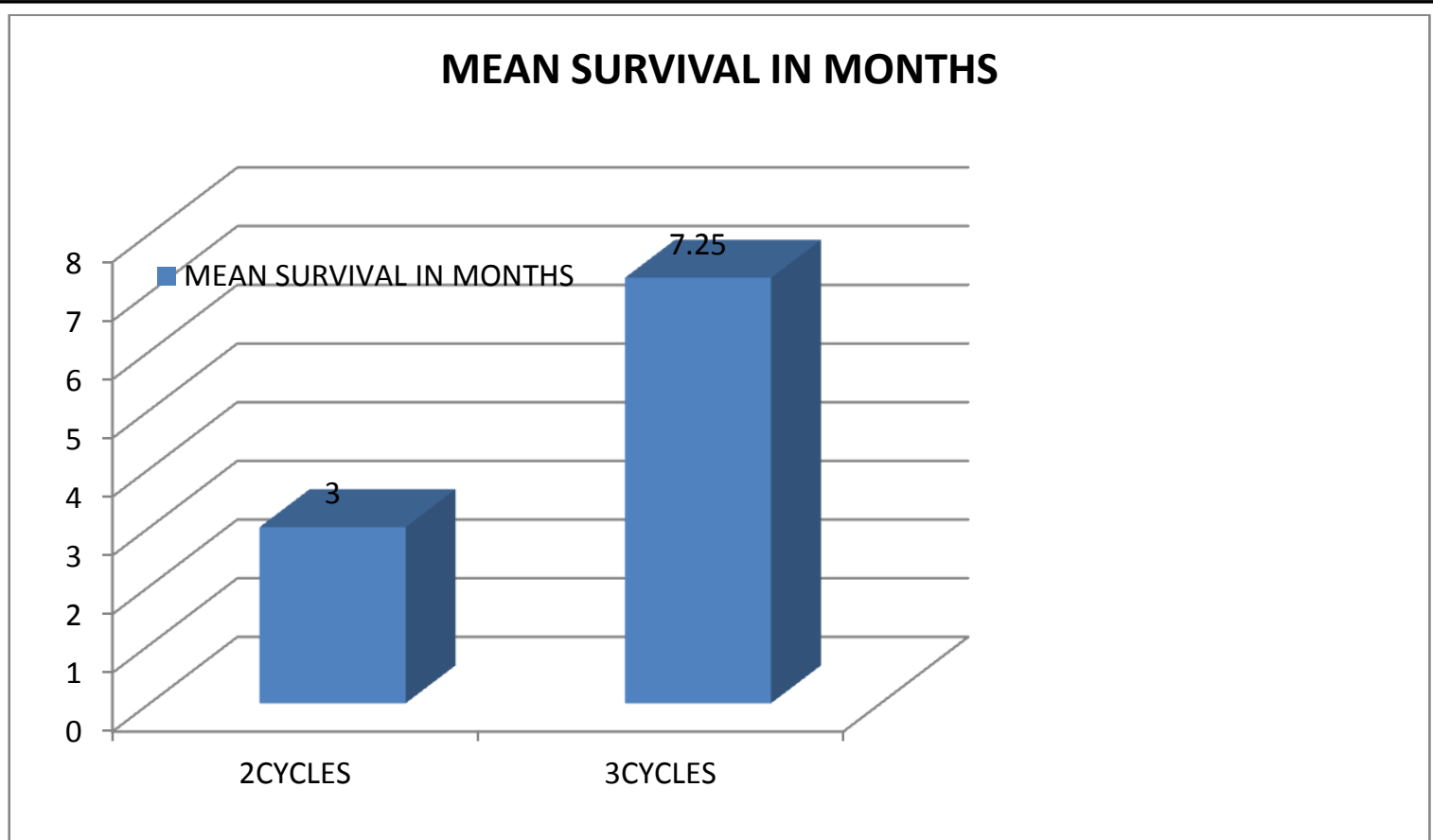

Figure 11 Comparison of duration of survival among those received 2 cycles and those received 3 cycles

\section{Kaplan Mayer Survivability function}

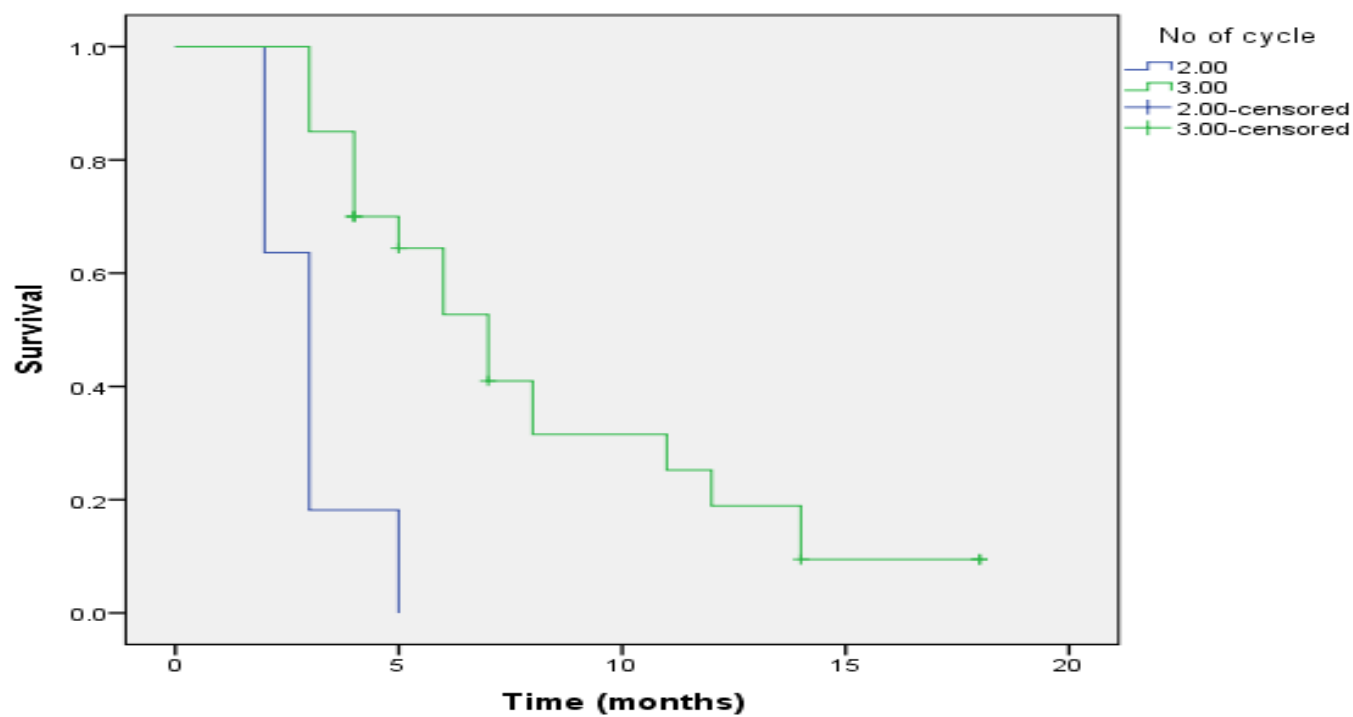

Figure 12 Kaplan Mayer Survivability function

Table 6 : Means and Medians for Survival Time

\begin{tabular}{|c|c|c|c|c|c|c|c|c|}
\hline \multirow[t]{3}{*}{ No of cycle } & \multicolumn{4}{|c|}{ Mean $^{a}$} & \multicolumn{4}{|c|}{ Median } \\
\hline & \multirow[t]{2}{*}{ Estimate } & \multirow[t]{2}{*}{ Std. Error } & \multicolumn{2}{|c|}{$95 \%$ Confidence Interval } & \multirow[t]{2}{*}{ Estimate } & \multirow[t]{2}{*}{ Std. Error } & \multicolumn{2}{|c|}{$95 \%$ Confidence Interval } \\
\hline & & & Lower Bound & Upper Bound & & & Lower Bound & Upper Bound \\
\hline 2.00 & 3.000 & .330 & 2.353 & 3.647 & 3.000 & .256 & 2.499 & 3.501 \\
\hline 3.00 & 8.085 & .778 & 6.561 & 9.610 & 7.000 & .701 & 5.625 & 8.375 \\
\hline Overall & 6.966 & 679 & 5.636 & 8.297 & 6.000 & .800 & 4.431 & 7.569 \\
\hline
\end{tabular}

a. Estimation is limited to the largest survival time if it is censored. 


\section{Weight gain}

Weight gain did not show any statistically significant difference between 2 groups Comparison of post test scores of the patients who received 2 cycles and those who received 3 cycles were done by using Analysis of covariance with before treatment values as covariate. This is done for making the initial value same for both group and then adjusting the post test value accordingly. Adjusted group means were then compared. Adjusted group mean along with the results of ANCOVA were given in Table 2.

The software used was SPSS Version 21.0

\section{Discussion}

Majority of the patients were 50 to 60 years. Gender wise males contributed to $66.7 \%$ and females $33.3 \%$ of the study population. Stagewise $52.9 \%$ were stage IVA disease, $45.1 \%$ had stage IVB disease and $2 \%$ had stage IV C disease. Histopathologically squamous cell carcinoma constituted 98\%, mucoepidermoid cell carcinoma $2 \% .70 .6 \%$ of the study population had an ECOG performance status of 1 and 29.4\% had an ECOG performance status of 2.40 patients completed 3 cycles of radiation. 11 patients took only 2 cycles of radiation. ECOG score remained same as preradiation level for patients who completed 3 cycles of radiation ECOG performance status of 2 patients (4\%) deteriorated following 2 cycles of radiation. Palliative response and quality of life showed significant improvement after each cycle of radiation Patients who completed 3 cycles of radiation got better palliative response when compared with those who took 2 cycles of radiation. $86 \%$ of the study population had symptomatic relief of pain. Trismus improved with each cycle of radiation.85\% of patients had symptomatic response to trismus. Dysphagia was a presenting symptom in $>95 \%$ of study population. Patients who had 3 cycles of radiation had better palliative response to dysphagia. Bleeding improved with each cycle of radiation. Complete response was seen after 3 cycles of radiation. Epistaxis was present in $7.8 \%$ of the study group. $50 \%$ of these patients had complete palliative response to radiation Toxicity assessment using RTOG Toxicity criteria showed grade 3 skin toxicity(pitting edema) in only $2 \%$ of the study group. Grade 3 mucosal toxicity seen in $2 \%$ of the study population. Overall grade 3 toxicity was seen in only $4 \%$ of the study population. Tumor response assessment after radiation showed complete response in $13.7 \%$ of total study group, partial response in $54.9 \%$, progressive disease in $7.8 \%$ respectively and in $23.5 \%$ the disease remained static. Quality of life assessment before and after each cycle of radiation revealed improvement in global health status with each cycle of radiation, Patients who completed 3cycles of radiation had better quality of life compared to those who completed 2 cycles. Functional scales including physical functioning, role functioning, emotional functioning, Cognitive functioning and social functioning showed significant improvement after radiation...Median overall survival was 5 months. Patients receiving 3 cycles of radiation had better palliative response and tumor response compared to those completing 2 cycle

\section{Conclusion}

Palliative hypo fractionated radiotherapy using RTOG 8502 regimen (quad shot) is an effective palliative treatment modality in locally advanced inoperable head and neck cancer. With excellent palliative response, radiobiological effect sparing normal tissue toxicity and improved overall survival it offers a better quality of life at low cost, best suited for centers with crowded population and poor patient compliance.

\section{References}

1. Jemal A, Bray F, Center MM,Ferlay J, Ward E, Forman D. Global cancer statistics.CA Cancer J Clin2011;61(2):6990

2. Sankaranarayanan R, Masuyer E, Swaminathan R, Ferlay J, Whelan S. Head and neck cancer: A global perspective on 
epidemiology and prognosis. Anticancer Res 1998;18:4779-86.

3. Chaturvedi AK, Engels EA, Anderson WF, Gillison ML: Incidence trends for(2)

a. human papillomavirus-related and unrelated oral squamous cell carcinomas in the United States, J Clin Oncol 26(4):612619, 2008.

4. Agarwal JP, Nemade B, Murthy V, et al. Hypofractionated, palliative radiotherapy for advanced head and neck cancer. Radiother Oncol: J Eur Soc Ther Radiol Oncol Oct 2008;89(1):51-6

5. Spanos Jr WJ, Clery M, Perez CA, et al. Late effect of multiple daily fraction palliation schedule for advanced pelvic malignancies (RTOG 8502). Int J Radiat Oncol, Biol, Phys 1994;29(5):961-7

6. Spanos Jr W, Guse C, Perez C, Grigsby P, Doggett RL, Poulter C. Phase II study of multiple daily fractionations inthe palliation of advancedpelvic malignancies: preliminary report of RTOG 8502. Int $\mathbf{J}$ Radiat Oncol, Biol, Phys Sep 1989;17 (3):659-61.

7. Paris KJ, Spanos Jr WJ, Lindberg RD, Jose B, Albrink F. Phase I-II study of multiple dailyfractions for palliation of advanced head and neck malignancies. Int J Radiat Oncol, Biol, Phys 1993;25(4):657-60.

8. Carrascosa LA, Yashar CM, Paris KJ, Larocca RV, Faught SR, Spanos WJ. Palliation of advanced pelvic malignancies: preliminary report of RTOG 8502. Int J Radiat Oncol, Biol, Phys Sep 1989; 17 (3):659-61. 